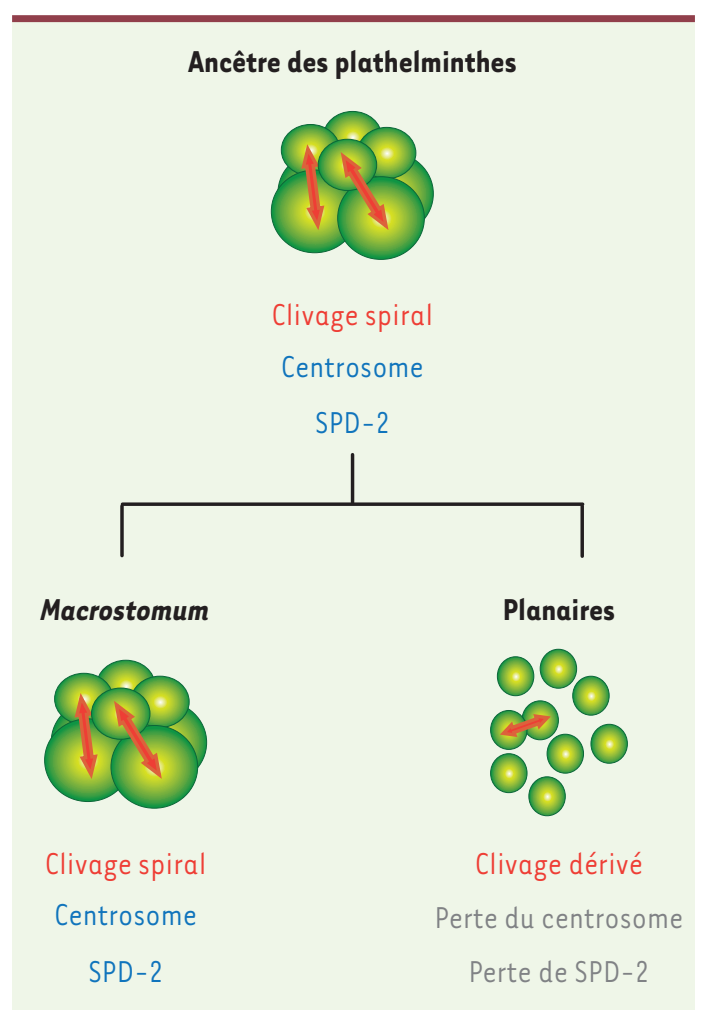

Figure 2. Modèle expliquant la perte du centrosome chez les planaires. Notre hypothèse est que la perte du centrosome est liée à un changement dans le mode de développement embryonnaire qui s'est produit au cours de l'évolution des plathelminthes. L'ancêtre des plathelminthes, ainsi que les plathelminthes qui se situent à la base de l'arbre phylogénétique, comme Macrostomum, utilisent un mode de clivage embryonnaire appelé clivage spiral. Le clivage spiral nécessite un contrôle précis de l'orientation des plans de divisions cellulaires, et donc a priori la présence de centrosomes. Chez les planaires, le développement n'implique pas de divisions en orientation contrôlée, et le centrosome a été éliminé.

organite déjà présent chez nos ancêtres unicellulaires, semble donc être essentiellement corrélée chez les animaux à des processus de développement, et pas seulement à des fonctions strictement confinées à chaque cellule.

L'étude des planaires nous renseigne donc, non seulement sur l'assemblage des centrioles et des cils affectés dans nombre de ciliopathies, mais aussi sur la fonction, la composition et l'évolution du centrosome, précisément parce que ce dernier a été perdu au cours de l'évolution des planaires. $\Delta$

What planarians tell us about cilia, centrioles and centrosomes

NOUVELLE

\section{Une altération de la neurogenèse conduit à des troubles de l'olfaction dans la sclérose en plaques}

Vanja Tepavčević, Brahim Nait-Oumesmar, Anne Baron-Van Evercooren
LIENS D'INTÉRÊT

Les auteurs déclarent n'avoir aucun lien d'intérêt concernant les données publiées dans cet article.

\section{REMERCIEMENTS}

L'auteur remercie Nicolas Borghi pour ses commentaires sur le manuscrit. Ce travail a été soutenu par le National Institutes of Health (NIH) et la Fondation W.M. Keck.

\section{RÉFÉRENCES}

1. Goetz SC, Anderson KV. The primary cilium: a signalling centre during vertebrate development. Nat Rev Genet 2010 ; 11 : 331-44.

2. Nigg $\varepsilon A$, Raff JW. Centrioles, centrosomes, and cilia in health and disease. Cell $2009 ; 139: 663-78$.

3. Azimzadeh J, Marshall WF. Building the centriole. Curr Biol $2010 ; 20$ : R816-25.

4. Wagner DE, Wang $I \varepsilon$, Reddien PW. Clonogenic neoblasts are pluripotent adult stem cells that underlie planarian regeneration. Science 2011 ; 332 : 811-6.

5. Newmark PA, Reddien PW, Cebria F, Sanchez Alvarado A. Ingestion of bacterially expressed double-stranded RNA inhibits gene expression in planarians. Proc Natl Acad Sci USA 2003; 100 : 11861-5

6. Andersen JS, Wilkinson CJ, Mayor T, et al. Proteomic characterization of the human centrosome by protein correlation profiling. Nature $2003 ; 426: 570-4$.

7. Azimzadeh J, Wong ML, Downhour DM, et al. Centrosome loss in the evolution of planarians. Science 2012 ; 335 : 461-3.

8. Tyler S. Development of cilia in embryos of the turbellarian Macrostomum. Hydrobiologia 1981 ; $84: 231-9$

9. Pereira G, Yamashita YM. Fly meets yeast: checking the correct orientation of cell division. Trends Cell Biol $2011 ; 21: 526-33$.

10. Cardona A, Hartenstein V, Romero R. Early embryogenesis of planaria: a cryptic larva feeding on maternal resources. Dev Genes Evol 2006 ; 216 : 667-81.
Centre de recherche de l'institut du cerveau et de la moelle épinière (Cricm), université Pierre et Marie Curie (UPMC) Paris 6, UMR-S975, Inserm U975, CNRS UMR 7225, assistance publique-hôpitaux de Paris (AP-HP), hôpital Pitié-Salpêtrière, 47, boulevard de l'Hôpital, 75013 Paris, France.

anne.baron@upmc.fr

[1] et participent, directement ou indirectement, à la ségrégation spatiale des réseaux neuronaux [2]. Les cellules souches de la ZSV assurent le renouvellement des interneurones et neurones périglomérulaires du bulbe olfactif dont
Les cellules souches du gyrus denté et de la zone sous-ventriculaire Ces dernières décennies ont été marquées par la découverte de cellules souches (CS) dans certaines régions du cerveau adulte: le gyrus denté et la zone sous-ventriculaire (ZSV). Ces cellules donnent naissance, tout au long de la vie, à des neurones aux fonctions particulières: les neurones issus des cellules souches du gyrus denté s'intègrent dans les circuits propres à cette structure 
la fonction principale est d'assurer la mémoire olfactive à long terme $[3,4]$.

La ZSV est composée de plusieurs types cellulaires définis selon des critères immunohistologiques et ultrastructuraux. Les cellules souches de cette niche (dites cellules B) expriment la GFAP (glial fibrillary acidic protein) et contactent la lumière du ventricule avec leur cil primaire [5]. Elles prolifèrent lentement et engendrent les cellules de précurseurs intermédiaires (type C) qui, eux, se divisent rapidement et se différencient ensuite en neuroblastes (cellules de type A). Ces derniers migrent au sein de la voie rostrale de migration (VRM) vers le bulbe olfactif où ils donnent naissance aux neurones granulaires et périglomérulaires [6]. Alors que la genèse des neurones du bulbe olfactif constitue la fonction primordiale de la ZSV dans le cerveau adulte, des études de traçage cellulaire démontrent que les cellules B sont aussi à l'origine d'une population d'oligodendrocytes dans la substance blanche périventriculaire. La balance entre neurogenèse et oligodendrogenèse dans le cerveau post-natal et adulte serait gouvernée par l'interaction de déterminants neurogéniques tels que les facteurs DIx2 (distal-less homeobox 2), Pax6 (paired box protein 6), et Olig2 (oligodendrocyte lineage transcription factor) qui régulent la genèse des oligo-

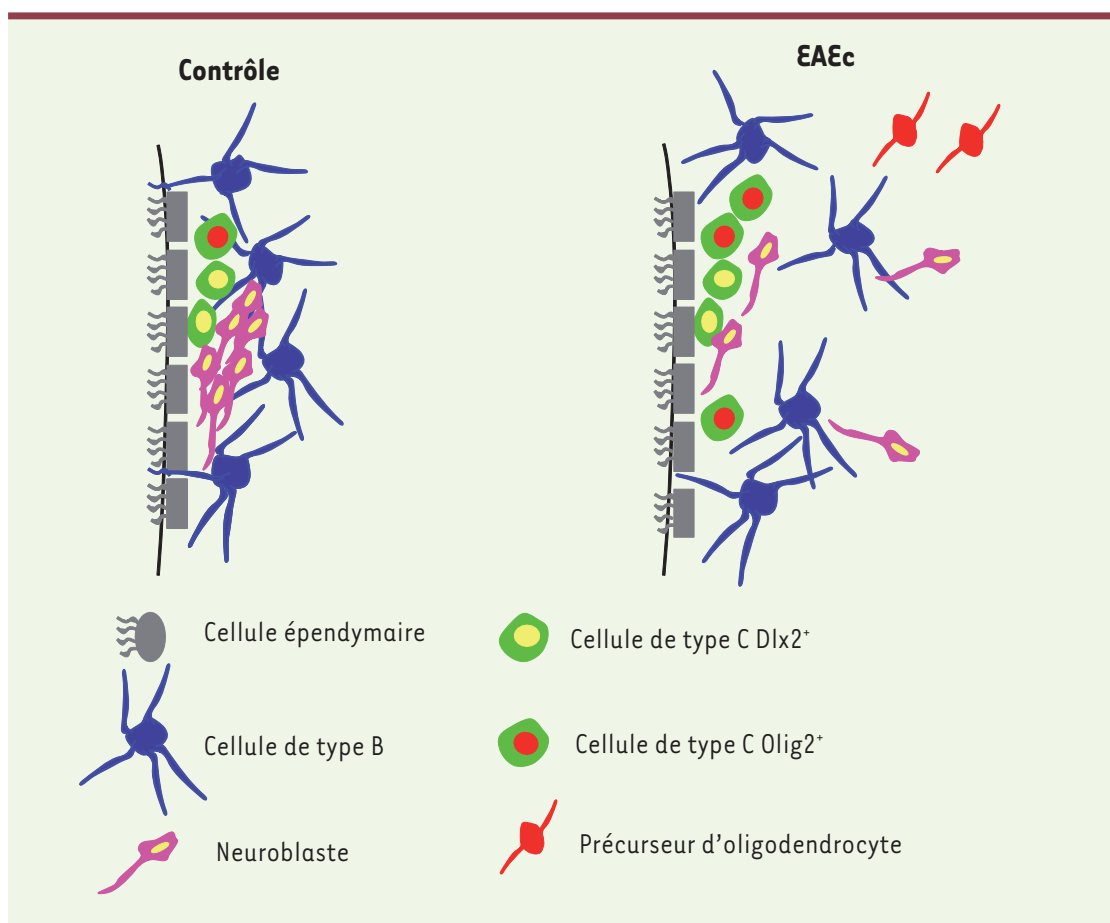

Figure 1. La zone sous-ventriculaire (ZSV) est modifiée au cours de l'EAE ciblée (EAEc). Dans le cerveau sain de souris adultes (contrôle, panneau de gauche), la niche de cellules souches est adjacente à la couche de cellules épendymaires multiciliées (gris). Les cellules souches primaires de la ZSV (cellules Bl) sont des astrocytes (en bleu) qui sont en contact avec le ventricule par un cil unique. Les cellules $B l$ prolifèrent lentement et génèrent les cellules de type $C$ à prolifération rapide (vert). Une sous-population de cellules C exprimant Dlx2 (cellules vertes à noyau jaune) prédomine par rapport à la sous-population de cellules $C$ exprimant Olig2 (cellules vertes à noyau rouge) et donne naissance aux neuroblastes (rouge) qui migrent vers le bulbe olfactif. Dans la ZSV d'EAEc ( $E A \varepsilon c$, panneau de droite), le nombre de cellules $B$ contactant le ventricule est réduit. La population de cellules C Olig2-positives augmente alors que le nombre de cellules C Dlx2positives demeure inchangé. Ceci conduit à une augmentation du nombre de cellules oligodendrogliales aux dépens du nombre de neuroblastes migrant vers le bulbe olfactif. De plus, certains neuroblastes migrent de façon ectopique dans le striatum et corpus calleux.

dendrocytes, et dans certains cas, celle des astrocytes $[7,8]$.

\section{Fonctions potentielles}

\section{des cellules souches de la ZSV}

\section{dans la sclérose en plaques}

Alors que dans le cerveau sain, les cellules souches ont un destin essentiellement neuronal, un certain nombre de travaux démontrent qu'elles ont la possibilité de se différencier vers d'autres types cellulaires (oligodendrocytes et astrocytes) en réponse à différentes situations lésionnelles [9]. Une telle plasticité pourrait être utile à des tentatives de réparation, mais ses conséquences sur le fonctionnement normal de la niche et sa contribution à des déficits neurologiques demeuraient jusqu'alors inconnues.

Au cours de ces dernières années, notre laboratoire s'est intéressé au potentiel de différenciation en oligodendrocytes des cellules de la ZSV dans des situations de démyélinisation. Les lésions de démyélinisation périventriculaires sont fréquentes dans la sclérose en plaques (SEP), une pathologie inflammatoire démyélinisante dont la durée est bien souvent associée à un échec de remyélinisation. La présence d'une niche de cellules souches à proximité de ces lésions pourrait constituer une source cellulaire d'intérêt pour la remyélinisation. En effet, l'induction de lésions de démyélinisation dans la zone périventriculaire augmente la prolifération des cellules de la ZSV et induit leur migration ectopique vers les lésions de démyélinisation où elles se différencient en oligodendrocytes $[10,11]$.

En dépit de ces observations expérimentales, la question de l'intégrité de la niche des cellules souches en situation de démyélinisation chronique inflammatoire reste débattue [11, 12], et les conséquences d'une altération de la niche sur le renouvellement des neurones du bulbe olfactif et l'olfaction restaient largement inexplorées. Par ailleurs, la fonction exacte de la ZSV chez l'homme est encore floue 
[13, 14]. En particulier, sa contribution à une neurogenèse bulbaire est encore discutée chez l'homme [15]. Les lésions de démyélinisation périventriculaire sont fréquentes dans la SEP et l'étude de la ZSV à proximité des lésions suggère, comme chez le rongeur, une augmentation de l'oligodendrogenèse et une migration ectopique de précurseurs gliaux vers les lésions $[16,17]$. Mais les conséquences de ces altérations sur la neurogenèse et les déficits olfactifs rencontrés chez les patients atteints de SEP [18] restaient à démontrer. Ces troubles olfactifs dans la SEP se caractérisent par des déficits de la reconnaissance olfactive n'affectant pas la sensibilité de détection.

\section{Rôle des cellules souches de la ZSV en situation de démyélinisation} Notre hypothèse est qu'une pathologie démyélinisante semblable à la SEP peut

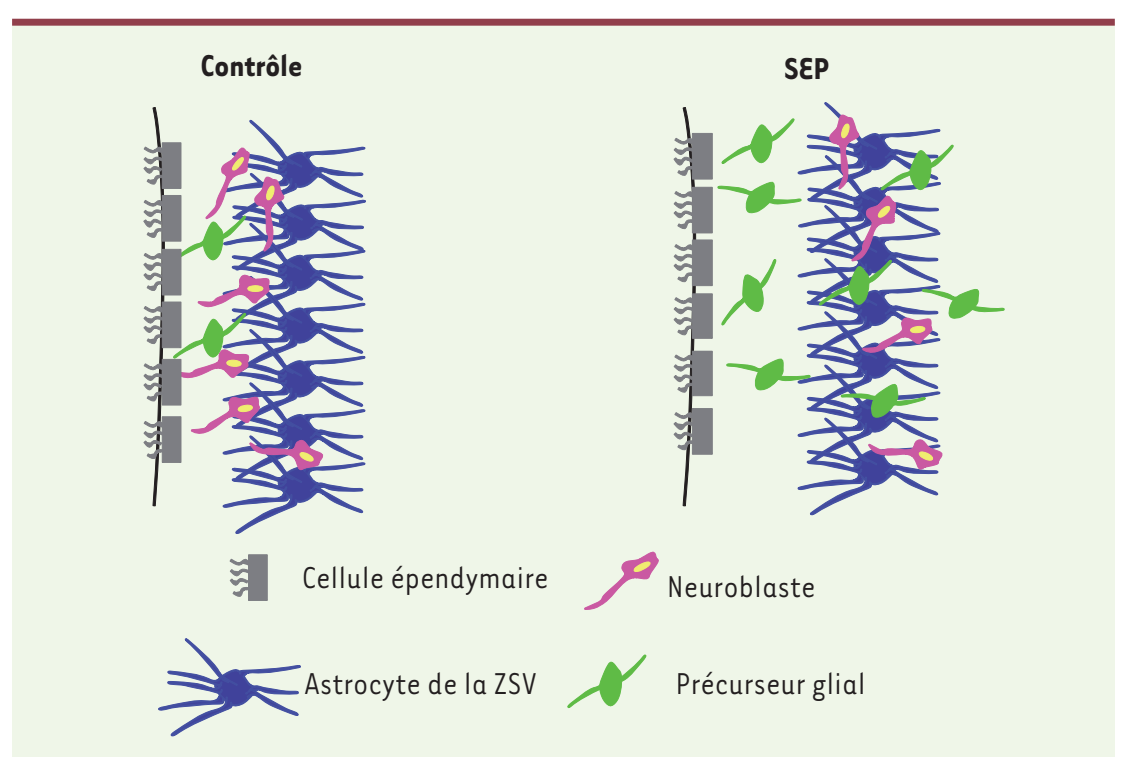

Figure 2. La ZSV change également chez les patients atteints de SEP. Une des différences majeures entre I'architecture de la ZSV humaine et celle de souris est la présence de régions cellulaires bien définies. II s'agit de la couche de cellules épendymaires (gris), une zone hypocellulaire (entre l'épendyme [gris] et les astrocytes [bleus]), le ruban astrocytaire (bleu), et la zone de transition adjacente au parenchyme. Chez les contrôles sans pathologies neurologiques, les neuroblastes (rouge) et quelques progéniteurs gliaux (vert) sont localisés dans la zone hypocellulaire et, plus rarement, dans le ruban astrocytaire. Dans la ZSV de cerveau SEP, le nombre de cellules dans la zone hypocellulaire augmente et comprend principalement des progéniteurs gliaux précoces, alors que le nombre de neuroblastes diminue. De plus, les neuroblastes et progéniteurs gliaux précoces se situent principalement dans et au-delà du ruban astrocytaire, ce qui suggère leur migration ectopique dans le parenchyme.
Ce modèle ne permet donc pas de suivre d'une façon précise les modifications survenant dans la ZSV en réponse à l'inflammation [17].

Afin de contourner cet écueil, nous avons développé un protocole d'EAE ciblée (EAEc) dans le corps calleux de souris C57/BI6, en nous inspirant d'un modèle développé dans la moelle épinière de rat [19]. Ce protocole consiste à immuniser des souris de façon subclinique à l'aide du peptide MOG (myelin oligodendrocyte glycoprotein) et d'adjuvant de Freund, puis d'injecter dans le corps calleux un cocktail de cytokines pro-inflammatoires (transforming growth factor- $\alpha$ [TNF $\alpha]$ et interféron- $\gamma[$ IFN $\gamma]$ ) permettant d'y attirer les cellules inflammatoires. Le déroulement temporel de la pathologie chez les souris atteintes d'EAEc associe successivement : une inflammation de l'encéphale avec des infiltrats inflammatoires dans le corps calleux, une phase de démyélinisation et une atteinte axonale, suivie d'une phase de remyélinisation. Ce modèle présente plusieurs avantages pour l'étude de la ZSV : (1) la présence de lésions inflammatoires périventriculaires chez tous les animaux, suivies d'une démyélinisation dans cette même localisation ; (2) l'injection de cytokines induit sans équivoque la pathologie dans le cerveau de tous les animaux, permettant ainsi une étude séquentielle précise de la dynamique des altérations de la ZSV. Nous avons analysé les modifications cellulaires et structurales de Ia ZSV par immunohistochimie et microscopie électronique, ainsi que la prolifération et le destin cellulaires par des études d'incorporation de BrdU (bromodéoxyuridine) (Figure 1). Nos résultats démontrent que le nombre de cellules inflammatoires fluctue au cours de la pathologie : il augmente rapidement au début de la maladie ( ${ }^{\text {re }}$ semaine) et s'accompagne d'une diminution transitoire de la prolifération dans la ZSV et d'une déplétion des neuroblastes. De plus, le nombre de cellules B (cellules souches) - qui 
contactent le liquide céphalorachidien des ventricules latéraux avec leur cil unique -, diminue alors que celui des cellules C (précurseurs intermédiaires) demeure inchangé. Après cette phase initiale, la prolifération des cellules de la ZSV conduit à une amplification des cellules $B$ et $C$, mais ne permet pas de corriger le déficit en neuroblastes chez les animaux atteints d'EAEc comparés aux animaux contrôles. Par ailleurs, les neuroblastes de la ZSV migrent de façon ectopique dans le corps calleux et le striatum. Au cours des phases chroniques tardives, on observe une persistance de cellules inflammatoires, une perte de neuroblastes et une augmentation des cellules de type C. Afin de mieux appréhender les mécanismes à l'origine de la déplétion de neuroblastes dans la ZSV en réponse à l'EA $\varepsilon c$, nous avons émis l'hypothèse que les cellules de type $C$ qui, en conditions normales, génèrent principalement des neuroblastes, se différencient préférentiellement en oligodendrocytes dans ce modèle. L'analyse de l'expression de déterminants neurogéniques, tels que Dlx2, et oligodendrogéniques, tels que Olig2, par les cellules de type $C$ a démontré que l'EAEc était associée à une réduction de la fraction $D \mid \times 2^{+}$au profit de la population Olig2 ${ }^{+}$, indiquant un changement de leur destin cellulaire au cours de la maladie. De plus, notre étude montre que des modifications de la voie de signalisation BMP (bone morphogenic protein)/pSMAD seraient impliquées dans ce changement de la destinée des cellules de type C, l'expression de PSMAD étant fortement réduite dans la ZSV des animaux atteints d'EA $\varepsilon$ c.

\section{Cellules souches de la ZSV}

\section{et neurogenèse du bulbe olfactif}

À la suite de ces données, nous avons exploré les conséquences fonctionnelles des altérations observées dans la ZSV sur la neurogenèse au sein du bulbe olfactif. Des études de suivi cellulaire ont démontré que l'attaque inflammatoire initiale diminue de manière significative le nombre des cellules des grains nouvellement produites dans le bulbe olfactif de souris $E A \varepsilon c$, mais augmente le nombre d'oligodendrocytes dérivés de la ZSV dans le corps calleux. L'analyse immunohistochimique de la doublecortine, un marqueur de neurones immatures, montre aussi de façon frappante que pendant la phase chronique de la maladie (trois mois après induction), la neurogenèse bulbaire demeure perturbée. Afin de déterminer si toutes ces altérations de la ZSV pouvaient avoir des conséquences fonctionnelles sur l'olfaction, nous avons analysé la discrimination olfactive et la mémoire olfactive à court (30 $\mathrm{min}$ ) et long terme (40 jours) chez les animaux atteints d'EAEc. Deux tests distincts de discrimination d'odeurs n'ont pas permis d'établir l'altération de ces paramètres chez les souris $E A \varepsilon c$, même au cours de la phase chronique de la maladie. Quant à la mémoire olfactive, à court terme elle n'est pas affectée, mais à long terme elle est clairement affectée, corroborant d'autres observations indiquant que la mémoire olfactive à long terme est la fonction principale de la neurogenèse adulte $[3,4]$.

Pour examiner la validité expérimentale de nos résultats dans la pathologie humaine, nous avons recherché une altération de la neurogenèse dans la ZSV de patients atteints de SEP (Figure 2). L'analyse immunohistochimique de la doublecortine indique la présence de neurones immatures dans les ZSV de cerveaux de patients SEP et de sujets contrôles. Cependant, le nombre de neuroblastes est significativement réduit dans la ZSV de patients SEP comparé au nombre dans la ZSV de sujets non atteints. Nos études précédentes indiquaient une augmentation de progéniteurs gliaux dans cette même région [16]. Cela suggère que, comme dans le modèle d'EAદc chez la souris, l'inflammation démyélinisante proche de la ZSV induit chez l'homme un déséquilibre de la balance neurogenèse/gliogenèse dans la ZSV, avec un enrichissement en précur- seurs gliaux au détriment de la genèse de nouveaux neurones.

$\varepsilon$ n conclusion, le modèle d'EAદ ciblée dans la substance blanche périventriculaire chez la souris nous a permis d'établir plusieurs conclusions concernant la fonction de la ZSV.

- L'effet majeur d'une inflammation démyélinisante de type SEP est l'altération de la neurogenèse adulte qui conduit à une déplétion à long terme de la genèse des cellules des grains.

- Néanmoins, la genèse des oligodendrocytes en réponse à la démyélinisation périventriculaire n'est pas affectée par l'inflammation démyélinisante.

- L'altération de la neurogenèse au profit de l'oligodendrogenèse se produit principalement dans les cellules de type $C$, et semble impliquer la voie de signalisation BMP.

- Ces événements conduisent à des altérations de mémoire olfactive à long terme chez la souris mimant ainsi les déficits olfactifs observés chez les patients atteints de SEP.

Notre travail apporte une explication alternative aux déficits olfactifs observés chez les patients atteints de SEP, attribués jusqu'à présent aux lésions inflammatoires dans les régions fronto/ temporales $[18,20]$. Bien que d'autres éléments expérimentaux soient nécessaires pour valider cette hypothèse, nos résultats alimentent l'idée grandissante que le dysfonctionnement des cellules souches neurales adultes contribue à la pathogenèse cérébrale. $\diamond$

Olfactory dysfonction in multiple sclerosis: role for subventricular zone progenitors

\section{REMERCIEMENTS}

Ce travail a été réalisé avec le soutien de l'Inserm, les Fondations ELA, ENP (École des neurosciences de Paris), et ARSEP (Aide à la recherche sur la $S E P$ ). V. Tepavčević a reçu le soutien de l'UPMC et de la FRM (Fondation pour la recherche médicale), B. Nait-Oumesmar et A. Baron-Van Evercooren sont récipiendaires de contrats d'interface AP-HP (Fédération de neurologie, Hôpital Pitié-Salpêtrière, Paris). 


\section{LIENS D'INTÉRÊT}

Les auteurs déclarent n'avoir aucun lien d'intérêt concernant les données publiées dans cet article.

\section{RÉFÉRENCES}

1. Toni N, Laplagne DA, Zhao C, et al. Neurons born in the adult dentate gyrus form functional synapses with target cells. Nat Neurosci 2008 ; 11 : 901-7.

2. Clelland CD, Choi M, Romberg C, et al. A functional role for adult hippocampal neurogenesis in spatial pattern separation. Science $2009 ; 325: 210-3$.

3. Lazarini F, Mouthon M, Gheusi G, et al. Cellular and behavioral effects of cranial irradiation of the subventricular zone in adult mice. PLoS One $2009 ; 4$ : e7017.

4. Sultan S, Mandairon N, Kermen F, et al. Learningdependent neurogenesis in the olfactory bulb determines long-term olfactory memory. FASEB $2010 ; 24: 2355-63$.

5. Doetsch F, Caillé I, Lim D, et al. Subventricular zone astrocytes are neural stem cells in the adult mammalian brain. Cell 1999; $97: 703-16$.

6. Carleton A, Petreanu L, Lansford R, et al. Becoming a new neuron in the adult olfactory bulb. Nat Neurosci $2003 ; 6: 507-518$
7. Hack M, Saghatelyan A, de Chevigny A, et al. Neuronal fate determinants of adult olfactory bulb neurogenesis. Nat Neurosci $2005 ; 8: 865-72$.

8. Marshall C, Novitch B, Goldman J. Olig2 directs astrocyte and oligodendrocyte formation in postnatal subventricular zone cells. J Neurosci 2005 ; 25 : 7289-98.

9. Kazanis I. The subependymal zone neurogenic niche: a beating heart in the centre of the brain: how plastic is adult neurogenesis? Opportunities for therapy and questions to be addressed. Brain 2009 ; 132 : 2909-21.

10. Nait-Oumesmar B, Decker L, Lachapelle F, et al. Progenitor cells of the adult mouse subventricular zone proliferate, migrate and differentiate into oligodendrocytes after demyelination. Eur J Neurosci $1999 ; 11: 4357-66$

11. Picard-Riera N, Decker L, Delarasse C, et al. Experimental autoimmune encephalomyelitis mobilizes neural progenitors from the subventricular zone to undergo oligodendrogenesis in adult mice. Proc Natl Acad Sci USA 2002 ; 99 : 13211-6.

12. Pluchino S, Muzio L, Imitola J, et al. Persistent inflammation alters the function of the endogenous brain stem cell compartment. Brain 2008; 131 : 2564-78.

13. Curtis M, Kam M, Nannmark U, et al. Human neuroblasts migrate to the olfactory bulb via a lateral ventricular extension. Science 2007 ; 315 : 1243-9.
14. Sanai N, Tramontin A, Quiñones-Hinojosa A, et al. Unique astrocyte ribbon in adult human brain contains neural stem cells but lacks chain migration. Nature $2004 ; 427: 740-4$

15. Bédard A, Parent A. Evidence of newly generated neurons in the human olfactory bulb. Res Dev Brain Res $2004 ; 151: 159-68$.

16. Nait-Oumesmar B, Picard-Riera N, Kerninon C, et al. Activation of the subventricular zone in multiple sclerosis: evidence for early glial progenitors. Proc Natl Acad Sci USA 2007 ; 104 : 4694-9.

17. Wu C, Chang A, Smith M, et al. Beta4 tubulin identifies a primitive cell source for oligodendrocytes in the mammalian brain. J Neurosci 2009 ; 29 : 7649-57.

18. Demarquay G, Ryvlin P, Royet J. Olfaction and neurological diseases: a review of the literature. Rev Neurol (Paris) 2007 ; $163: 155-67$.

19. Kerschensteiner M, Stadelmann C, Buddeberg B, et al. Targeting experimental autoimmune encephalomyelitis lesions to a predetermined axonal tract system allows for refined behavioral testing in an animal model of multiple sclerosis. Am J Pathol 2004 ; 164 : 1455-69.

20. Doty R, Li C, Mannon L, Yousem D. Olfactory dysfunction in multiple sclerosis. Relation to plaque load in inferior frontal and temporal lobes. Ann NY Acad Sci 1998 ; 855 : 781-6.

\section{NOUVELl $\varepsilon$}

\section{Implications du gène d'horloge Reverb $\alpha$ dans l'obésité}

Département neurobiologie des rythmes, Institut des neurosciences cellulaires et intégratives, CNRS UPR 3212 associé à l'université de Strasbourg, 5, rue Blaise Pascal, 67084 Strasbourg, France.

challet@inci-cnrs.unistra.fr
$>$ En raison de sa prévalence sans cesse croissante, l'obésité, que l'on peut définir comme un excès de masse adipeuse, est devenue un problème majeur de santé publique, dans la mesure où la surcharge adipeuse est associée à de nombreuses complications métaboliques, telles que le diabète, l'hypertension et l'athérosclérose. Les facteurs génétiques mis à part, les deux causes principales d'obésité sont une surconsommation d'aliments riches en calories et un mode de vie sédentaire $[1,2]$. Caractérisée plus récemment, la désorganisation temporelle est un facteur aggravant qui favorise les désordres métaboliques [3]. D’où la nécessité de prendre en considération la rythmicité journalière de la prise alimentaire et du métabolisme énergétique.

\section{Le concept de «chronobésité 》}

Des horloges moléculaires dans le cerveau et les organes périphériques régulent notre physiologie sur $24 \mathrm{~h}$. Ces variations endogènes, appelées rythmes circadiens (du latin circa: environ, et dies : jour), permettent aux organismes d'anticiper et d'être préparés aux changements périodiques de l'environnement, incluant le cycle lumière-obscurité et la disponibilité en nourriture. Or, des conditions inhabituelles d'éclairement (exposition à la lumière au cours de la nuit) ou des repas pris à des heures inadaptées pour l'organisme (pendant les heures normalement consacrées au sommeil) sont à la fois des situations de désynchronisation (c'est-à-dire qui altèrent la rythmicité circadienne) et des facteurs de risque métabolique. Ces arguments nous ont conduits à définir le concept de « chronobésité », c'est-à-dire une surcharge lipidique causée par une désorganisation circadienne [4].

\section{Les protéines d'horloge}

Les rythmes circadiens sont produits par un réseau d'horloges endogènes, dont la principale est située dans les noyaux suprachiasmatiques de l'hypothalamus. L'horloge suprachiasmatique est surtout remise à l'heure par la lumière ambiante perçue par la rétine. Les autres horloges et oscillateurs secondaires sont largement distribués dans l'organisme : rétine et nombreuses régions cérébrales, la quasi-totalité des tissus périphériques, dont le foie, le pancréas et les tissus adipeux. Ces horloges secondaires sont, pour la plupart, très sensibles à l'effet synchroniseur des repas. 